\title{
Interplay between intra-urban population density and mobility in determining the spread of epidemics
}

\section{Surendra Hazarie}

University of Rochester

David Soriano Panos

University of Zaragoza https://orcid.org/0000-0002-6388-4056

\section{Alex Arenas}

Universitat Rovira i Virgili https://orcid.org/0000-0003-0937-0334

Jesus Gomez-Gardenes

University of Zaragoza https://orcid.org/0000-0001-5204-1937

Gourab Ghoshal ( $\square$ gghoshal@pas.rochester.edu )

University of Rochester https://orcid.org/0000-0001-7593-5626

\section{Article}

Keywords: population density, epidemics, SARS-COV-2, urban areas

Posted Date: February 5th, 2021

DOI: https://doi.org/10.21203/rs.3.rs-186291/v1

License: (c) (i) This work is licensed under a Creative Commons Attribution 4.0 International License.

Read Full License 


\title{
Interplay between intra-urban population density and mobility in determining the spread of epidemics.
}

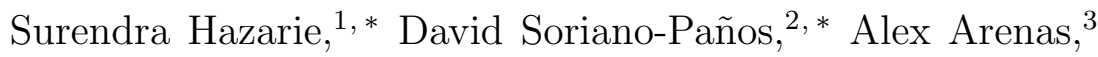 \\ Jesús Gómez-Gardeñes, ${ }^{2,4, \text { I }}$ and Gourab Ghoshal ${ }^{1,5}$, 周 \\ ${ }^{1}$ Department of Physics \&3 Astronomy, \\ University of Rochester, Rochester, NY, 14627, USA. \\ ${ }^{2}$ GOTHAM Lab - Department of Condensed Matter Physics and \\ Institute for Biocomputation and Physics of Complex Systems (BIFI), \\ University of Zaragoza, E-50009 Zaragoza, Spain. \\ ${ }^{3}$ Departament d'Enginyeria Informàtica i Matemàtiques, \\ Universitat Rovira i Virgili, E-43007 Tarragona, Spain. \\ ${ }^{4}$ Center for Computational Social Science (CCSS), \\ Kobe University, Kobe 657-8501, Japan. \\ ${ }^{5}$ Department of Computer Science, University of Rochester, Rochester, NY, 14627, USA.
}

\begin{abstract}
In this work, we address the connection between population density centers in urban areas, and the nature of human flows between such centers, in shaping the vulnerability to the onset of contagious diseases. A study of 163 cities, chosen from four different continents reveals a universal trend, whereby the risk induced by human mobility increases in those cities where mobility flows are predominantly between high population density centers. We apply our formalism to the spread of SARS-COV-2 in the United States, providing a plausible explanation for the observed heterogeneity in the spreading process across cities. Armed with this insight, we propose realistic mitigation strategies (less severe than lockdowns), based on modifying the mobility in cities. Our results suggest that an optimal control strategy involves an asymmetric policy that restricts flows entering the most vulnerable areas but allowing residents to continue their usual mobility patterns.
\end{abstract}

\footnotetext{
* These two authors contributed equally to this work.

† gardenes@unizar.es

¥gghoshal@pas.rochester.edu
} 


\section{Introduction}

During the last century, humankind has rapidly evolved into an interconnected society driven by the existence of a vast mobility network connecting different areas around the globe. In particular, the striking growth experienced by the international mobility network [1] has helped to bridge socio-cultural [2 4] and economic gaps [5]. Accompanying this is the phenomenon of urbanization, whereby a majority of the world's population reside in densely packed urban centers, with the trend only accelerating [6, 7], given the socioeconomic advantages that cities afford [8, 9]. Allayed against these benefits, the increase in mobility has removed the main bottleneck limiting the spatial diffusion of confined epidemic outbreaks. Once the disease spreads to different regions, it takes advantage of the high population density and infrastructure networks in cities [10] to rapidly spread through the local population. As a consequence, over the past few years, several contagious disease outbreaks have emerged, notable among them H1N1 in 2009 [11], Ebola in 2014 [12, ZIKV in 2015 [13] and of course more recently SARS-COV-2 [14, 15]. Indeed, the frequency with which these pandemics occur is troublingly increasing [16].

Despite the different nature of the pathogens, their spreading, both globally and locally, is primarily explained by the sequential combination of case importation from contagion sources, followed by local community transmission converting initially unaffected regions into new endemic areas. Different flavors of mobility play a role in each process: on the one hand, long-haul flights [17] are usually the drivers facilitating the entry of pathogens into a given country, to the extent that the airport mobility network has proved to be a reliable proxy to determine pathogen arrival times [18, international infection routes [19], and to complement phylogeographic inference of emerging pathogens such as SARS-CoV-2 [20]. On the other hand, once index cases are found within a given region, a complex combination of the local mobility [21] and socio-economic features of the population [22, 23] determines the speed of epidemic spread and the extent of its outbreak.

Quantifying the impact of local mobility on the global diffusion of a pandemic constitutes a challenging task. In this sense, several examples addressing the impact of daily recur- 
rent mobility patterns on the spread of contagious diseases can be found in the literature [24 31]. The majority of these, however, are theoretical frameworks analyzing the features of synthetic mobility networks, and the influence of total volume of travelers on the course of the epidemic. Nonetheless, recent advances made in data-gathering techniques allow for obtaining accurate representations of daily urban rhythms constructed from mobile phone traces [32], geolocalized tweets [33], Location Based Social Networks [34], or extensive census surveys. These data sets enable the extension of the theoretical machinery to address real epidemic scenarios. Indeed, recurrent mobility patterns have been already useful for identifying the most exposed areas in some epidemic scenarios [35] as well as reproducing the infection routes of H1N1 influenza [36], Malaria [37], and more recently SARS-COV-2 [38 41].

While much attention has been spent on reconstructing past infections, or epidemic forecasting in the case of extant pandemics, an important question that immediately arises, is what makes regions - in particular urban agglomerations where most people reside - vulnerable to the spread of pathogens in the first place? While factors such as population density, levels of healthcare, quality of infrastructure and socioeconomic disparities play a major role [42], vulnerability to spread is a complex interplay between these features that is, in general, difficult to disentangle. For instance, the role of population density is an open question with evidence both for and against its influence on epidemic spreading [43, 44]. Indeed, merely the density of contacts, while relevant at a neighborhood level, is not enough to explain the mechanisms of spread; one would also need to consider the mobility network of flows that govern the exchange of people between the regions. In such a setting, the spatial distribution of the population densities and the strength of interaction between the regions become especially relevant.

In other words it is reasonable to assume that the morphology of the city in terms of how its residents are distributed and how they navigate the city plays a crucial role in their susceptibility to pandemics. Indeed, recent studies have shown that the spatial patterns of how residents utilize transportation infrastructure is a strong indicator of that regions' levels of social inclusion, quality of infrastructure and wealth creation [45]. In [46] the authors 
propose a measure of a city's dynamical organization based on mobility hotspots [47] to classify them in a spectrum between compact-hierarchical and sprawled layouts. The extent to which cities are compact or sprawled serve as a low-dimensional proxy for various urban indicators related to quality of life, health and pollution.

In this work we connect the dots between the morphology of human activity in cities, in terms of its associated mobility flows and the distribution of resident populations, and its effect on shaping the transmission of infectious diseases and their associated epidemic outbreaks. We collect data from 163 cities across four continents, on their population density at the zip-code level, and intra-urban mobility flows for the first half of 2020. Using this we extract population density hotspots (i.e. those areas with the highest concentration of residents) and measure the extent to which flows between hotspots dominate the total flows in the city. To capture epidemic spreading, we generalize a MIR (Movement-Interaction-Return) epidemic model [27] that captures the interplay between recurrent mobility flows and the distributions of resident populations. We derive the epidemic threshold, representing the minimum infectivity per contact required to instigate an epidemic outbreak, and connect it with the distribution of flows among population density hotspots. In particular we show that, despite their ostensible differences in terms of spatial layout, evolutionary history, or levels of infrastructure, all considered cities lie on a universal curve capturing an inverse relationship between the epidemic threshold and the extent to which mobility flows are localized between hotspots.

The results suggests an increased susceptibility to epidemics as a function of flows being concentrated between high population centers. As a proof-of-concept, we analyze the current SARS-COV-2 pandemic by quantifying the epidemic growth from the initial infection curves as an empirical proxy for city vulnerability and plotting it against our calculated epidemic threshold. The empirical trends match our theoretical formalism where cities with mobility concentrated primarily between hotspots are more vulnerable as compared to those with more egalitarian mobility distributions. Based on this observation, we propose a realistic mitigation policy that, being much less severe than draconian lockdowns, lowers the susceptibility of cities that lie in the vulnerable spectrum. 


\section{Results}

\section{A. Data}

The population density for cities at zip-code resolution was collected from national census bureaus [48 50] and from high resolution population density estimates recently published by Facebook [51]. Each of these cities correspond to the largest in their respective countries in terms of population size. From this data, we extract $H_{k}$ population density hotspots for each city $k$ (varying from city-to-city) by applying a non-parametric method based on the derivative of the Lorenz curve [46, 47] (for details of the calculation see Supplementary Section S1).

The mobility flows within each city are sourced from the Google SARS-COV-2 Aggregated Mobility Research Dataset, and contain anonymized flows mobility flows aggregated over users who have turned on the Location History setting, which is off by default. The flows are between cells of approximately $5 \mathrm{~km}^{2}$ for the period ranging November 3rd 2019 to February 29th 2020. For the purposes of our analysis we only consider the period before any mobility mitigation measures were initiated as a response to the SARS-COV-2 pandemic (see Supplementary Section S2 for further details). The flows are encoded in a matrix $\mathbf{T}$ whose elements $T_{i j}$ correspond to the population out-flows from $i$ to $j$ and whose diagonal elements correspond to self-flows. For each city $k$, given $H_{k}$ hotspots, we calculate the hotspot concentration, $\kappa_{k}$, defined as the fraction of total flows in the city that occur only between hotspots thus,

$$
\kappa_{k}=\frac{\sum_{i, j \in H_{k}} T_{i j}}{\sum_{i, j} T_{i j}} .
$$

This metric lies in the range $0 \leq \kappa_{k} \leq 1$, with the limiting cases corresponding to flows exclusively between hotspots or only between hotspot and non-hotspot areas. The list of cities for each country, the administrative unit, and the hotspot concentration is shown in Tab. S1. The results show a wide spectrum of values $\left(0.05 \leq \kappa_{k} \leq 0.79\right)$ both within and between countries, indicating significant variability in cities in terms of the morphology of 
Adelaide $(\kappa=0.52)$

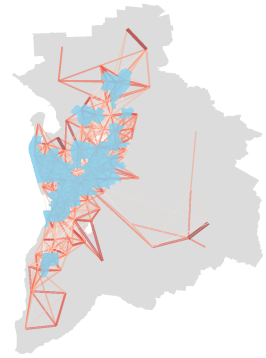

Detroit $(\kappa=0.46)$

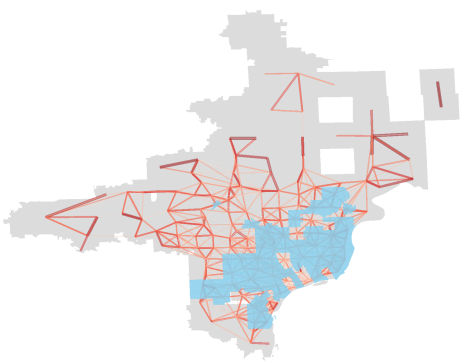

Melbourne $(\kappa=0.25)$

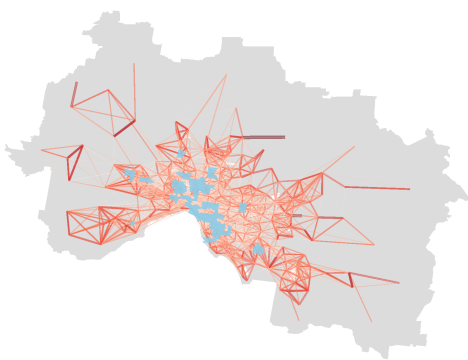

Chicago $(\kappa=0.18)$

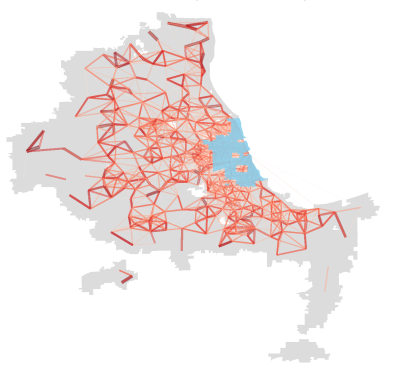

$10^{-1}$
Sydney $(\kappa=0.16)$

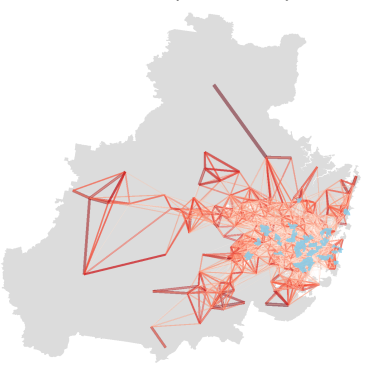

Miami $(\kappa=0.11)$

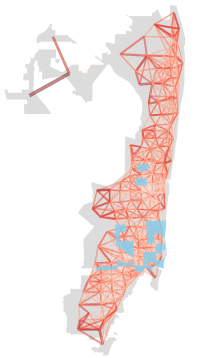

FIG. 1. Spatial representation of the urban mobility network for selected cities (upper panel) Autralia, (lower panel) United States. Areas marked blue correspond to population density hotspots. The cities are organized in descending order according to the extent to which mobility flows are concentrated between areas of high population density, $\kappa$ (Eq.(1)). Line color encodes the number of inhabitants following each route normalized by the highest flow observed within each city.

human flows between population centers.

In Fig. 1, we plot the spatial layout of the hotspots and the mobility network for six representative cities in Australia (upper panel) and the United States (lower panel). The cities are organized in descending order of $\kappa$, and it is apparent that in those cities with high $\kappa$, flows are mainly confined around hotspots (marked in red), whereas they are increasingly more distributed with decreasing $\kappa$. An additional feature is that hotspots are more spatially concentrated in those cities with lower $\kappa$ and dispersed in those with higher $\kappa$, indicating a more heterogeneous distribution of population density in the former as compared to the latter. 


\section{B. Model}

To characterize a city's vulnerability to disease spread, we calculate the epidemic threshold by generalizing the formalism introduced in [27]. In what is to follow, we incorporate a Susceptible (S), infectious (I) or recovered (R) dynamics, however, we note that the formalism is easily generalizable to more elaborate compartmental schemes. An infectious individual transmits the pathogen to healthy counterparts via direct interaction at a rate $\lambda$. In turn, infectious individuals enter the compartment $R$ at a rate $\mu$, which typically encodes the inverse of the expected contagious period. The mixing among healthy and infectious individuals is governed by the spatial distribution of the population and their mobility patterns. The different zip-codes of the city are represented as patches $i$, which are initially populated by $n_{i}$ residents. The activity of the residents is considered on a daily basis and split into three stages: Movement, Interaction and Return. Residents decide to either move to a different patch with probability $p$, or remain with probability $1-p$. If the former, the choice of location is proportional to the elements of the origin destination matrix $\mathbf{T}$. After all movements (or lack thereof) have been completed, interactions occur within patches according to a mean-field assumption where every individual makes the same number of contacts proportional to the population density via a function $f_{i}$; the final step involves return to the place of residence. The same process is then repeated for the next time-step (day).

Given $N$ patches in a city, the dynamics is completely specified by $2 \times N$ coupled discrete equations governing the temporal evolution of the fraction of infected and recovered individuals residing in each patch. Namely, the fraction of infected, $\rho_{i}^{I}(t+1)$, and recovered individuals, $\rho_{i}^{R}(t+1)$, associated to patch $i$ at time $t+1$, reads:

$$
\begin{aligned}
& \rho_{i}^{I}(t+1)=(1-\mu) \rho_{i}^{I}(t)+\left(1-\rho_{i}^{I}(t)-\rho_{i}^{R}(t)\right) \Pi_{i}(t) \\
& \rho_{i}^{R}(t+1)=\rho_{i}^{R}(t)+\mu \rho_{i}^{I}(t),
\end{aligned}
$$

where $\Pi_{i}(t)$ represents the probability of a susceptible individual resident in $i$ to contract the disease at time $t$. Assuming that the process reaches a steady-state and that the size of the 
outbreak is small in comparison to the overall population, after a sequence of manipulations (Supplementary Section S3, Eqns. S4-S15), it can be shown that the epidemic threshold is of the form, $\lambda_{c}(p)=\mu / \Lambda_{\max }(\mathbf{M})$, where $\Lambda_{\max }$ is the spectral radius of a mixing matrix $\mathbf{M}$, taking into account the mobility flows, the degree of mobility $p$, the effective population in a given patch, and the number of contacts as a function of population density in that patch. If $p=0$ then the threshold would correspond to a static population that never moves, whereas if $p=1$ then this accounts for a fully active population. Based on this observation one can define a normalized epidemic threshold $\tilde{\lambda}_{c}=\lambda_{c}(p=1) / \lambda_{c}(p=0)$ to focus only on mobility effects while removing the influence of the population density.

\section{Connecting the epidemic threshold to hotspot flows}

Given the quantitative description of human flows composing the mobility backbone of each city, we now focus on determining the effect that their morphology has on the epidemic threshold. Given the extensive list of considered cities, and the attendant variation in population density, in order for a fair characterization, it is important to remove this component, and therefore the relevant parameter here is the normalized threshold $\tilde{\lambda}_{c}$. In Fig. 2A we plot $\tilde{\lambda}_{c}$ as a function of $\kappa$ for all the cities in our dataset, represented as filled circles colored according to their respective countries. We find a universal trend, whereby all cities fall into a curve marking an inverse relationship between the vulnerability of the cities and the extent to which flows are concentrated between hotspots, i.e $\tilde{\lambda}_{c} \sim \kappa^{\beta}$ with $\beta \approx-0.25$. In partic-

ular, cities where there is a more egalitarian distribution of flows (lower $\kappa$ ), the epidemic threshold is higher for a mobile population as compared to a static population, indicating that movement between regions lowers the risk of an epidemic outbreak. Conversely, in those cities where the population moves primarily between hotspots, there is little-to-no difference in risk in terms of whether residents stay in their patches, or whether they move to different ones. In Fig. S1, we show the equivalent of Fig. 2/A but now split by country finding the same trend attesting to the robustness of the inverse dependence. (The fits to $\beta$ for each country are shown in Tab. S1). As a comparison to other population-related measures, in 

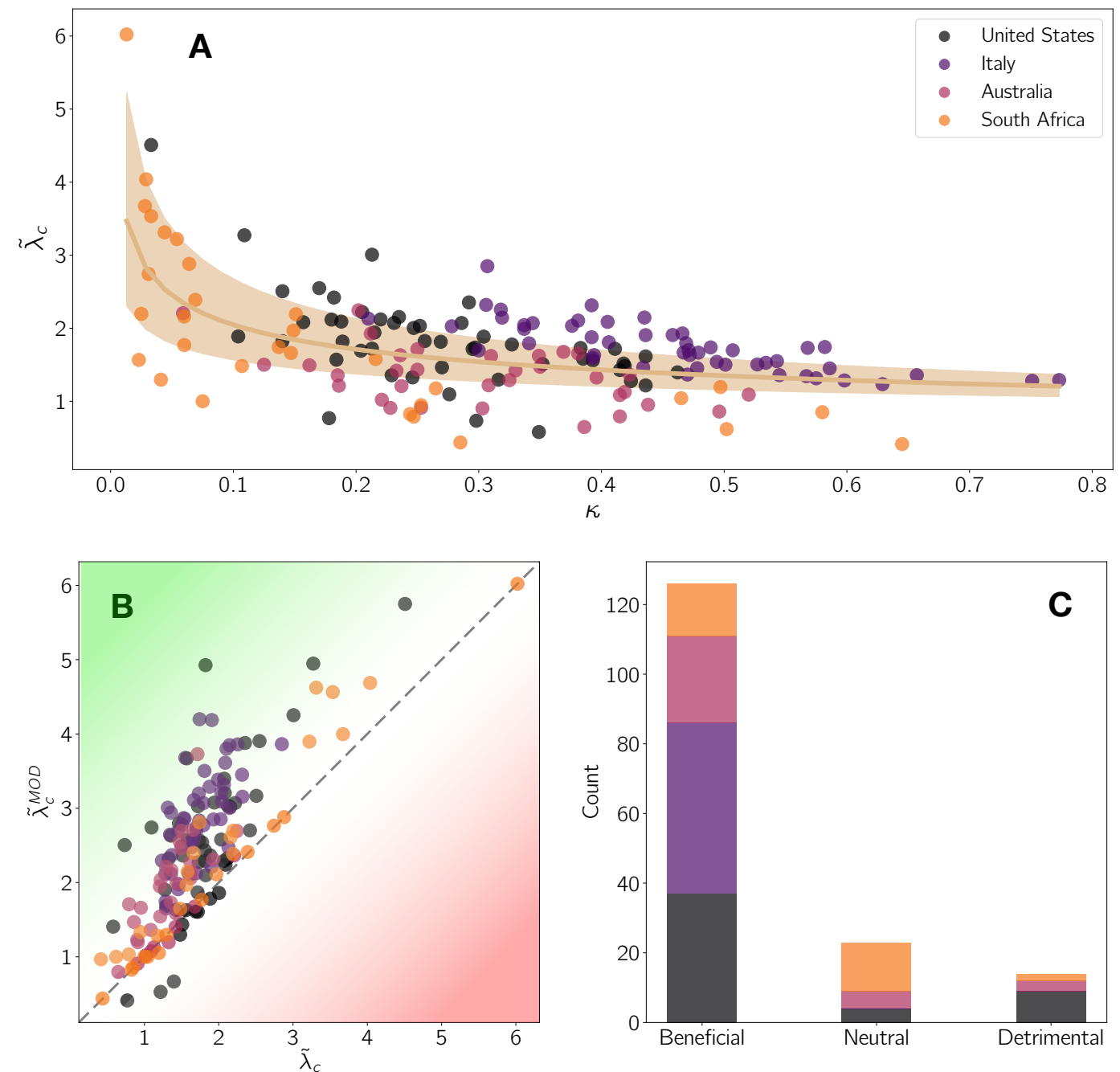

FIG. 2. Connecting morphology to vulnerability A Normalized epidemic threshold $\tilde{\lambda}_{c}$ versus population density hotspot concentration $\kappa$ for cities in each of the chosen 4 countries (color code). The solid line shows the fit to a power-law function given by $\tilde{\lambda}_{c}=A \kappa^{\beta}$, with $A=1.15 \pm 0.06$ and $\beta=-0.25 \pm 0.03$. The shadowed region covers the $95 \%$ confidence interval. $\mathbf{B}$ Normalized threshold after removing all the flows connecting hotspots $\tilde{\lambda}_{c}^{M O D}$ as a function of the normalized epidemic threshold without intervention $\tilde{\lambda}_{c}$. The green (red) areas contains those cities for which removing flows among hotspots is beneficial (detrimental). C Division of the cities according to the result: beneficial, neutral and detrimental as described in the text.

Fig. S2, we plot the Spearman correlation matrix for $\kappa$, the average population density, the Lloyd mean crowding [52] and $\tilde{\lambda}_{c}$ for the 50 American cities. The figure suggests that the concentration of flows between areas of high population density, $\kappa$, is a much better predictor of the vulnerability of cities than the other two measures. 
To further characterize the relation existing between cities' vulnerability and the concentration of mobility between density hotspots, we next analyze the impact of reshuffling the flows at a local scale for each of the analyzed cities. In particular, we preserve the total amount of flows, by removing all links connecting hotspots (thus setting $\kappa=0$ ) and redistributing them evenly across non-hotspot locations. We then recompute the resulting normalize threshold $\tilde{\lambda}_{c}^{M O D}$ and in Fig. $2 \mathrm{~B}$ plot it as a function of the threshold corresponding to the unperturbed network $\tilde{\lambda}_{c}$. As the figure indicates for the vast majority of cities (irrespective of their original value of $\kappa$ ), the effect of switching off the flows between hotspots leads to an increase in the epidemic threshold, in turn lowering their vulnerability to epidemic spread. In particular there appears to be three categories of cities: beneficial, those where the threshold is increased greater than 10\%; neutral, those where the threshold is increased by less than 10\%; and finally detrimental, those where the threshold is instead lowered. The number of cities belonging to each category is plotted as a bar-chart in Fig. $2 \mathrm{C}$, indicating that around $75 \%$ of cities experience a lowering of their vulnerability, $15 \%$ remain neutral, and the remaining $10 \%$ experience an increased susceptibility (interestingly this category is dominated by American cities). For the small number of cities, where we find this counterintuitive effect of lowered threshold, it is likely that there are other more complex features at play not considered in this analysis.

Nevertheless, these results provide overwhelming evidence that, in most cases, the concentration of human mobility between densely populated areas is a feature that enhances disease spreading and makes such cities vulnerable to epidemics. Moreover, the beneficial effect caused by the reorientation of intra-hotspot flows towards less densely populated areas seems to be rooted in a homogenization of the distribution of the underlying density, which, as mentioned in [28, 31], enforces infected individuals to stay away from the contagion focus, thus reducing their infection power. In turn, this homogenizing flow structure appears naturally in cities with low $\kappa$ and, as suggested by the empirical trends in Fig. 2 A, characterizes the most resilient cities. Therefore, a lower $\kappa$ translates into a greater mix of populations, between high and low population density centers, where they can actually take advantage of mobility between city sub-regions to prevent outbreaks. 


\section{Application to real pandemic settings}

The formalism proposed here can readily be applied to assess the exposure of cities to actual outbreaks. To illustrate this, we next focus on the spread of SARS-COV-2 in the 50 most populated Core-Based-Statistical-Areas (CBSA) in the United States, chosen due to the appropriate spatial resolution in terms of infection data. Note that, although, thus far, we have focused on the SIR model, the following will illustrate the generality of the results, in the context of the spread of SARS-CoV-2, that has been recently analyzed with more elaborate compartmental models [38, 52,55$]$.

As a proxy for a city's vulnerability to epidemic spread, we make use of the number of confirmed infected cases at the county-level collected from the New York Times [56] and USAFacts [57]. Given the inherent noise due to reporting artifacts, and assuming an exponential growth, $I_{k}(t) \sim \exp \left(b_{k} t\right)$ during early onset, we apply a smoothing procedure to extract the growth-rate $b_{k}$ of the number of infected cases, and use that as proxy for a city $k$ 's susceptibility to disease spread. To remove any effects due to non-pharmaceutical interventions and behavioral changes in the population, we focus on the period before mitigation measures. The full details of the procedure are shown in Supplementary Section S5, and the temporal infection plots for each city along with the fits are shown in Fig. S3.

In order to properly connect with the growth rate, we need to reintroduce the effect of the population distribution and, therefore, the relevant variable is the unnormalized threshold $\lambda_{c}(p=1)$. Note that, by choosing $p=1$, we force all the inhabitants within a city to follow the flow matrix $\mathbf{T}$, but not all of them leave their residential area due to existence of self-loops in this matrix. Indeed, according to the avalaible data for the cities analyzed here, around $36 \%$ of the population remains on average inside their residential administrative unit.

In Fig. 3A we plot the empirically extracted growth rate $b$ as a function of the epidemic threshold, $\lambda_{c}$, finding once again an inverse trend, confirming the role of the threshold as a proxy for vulnerability. Those cities which experienced a faster epidemic growth during the early onset of the pandemic indeed had a lower threshold according to our formalism. 

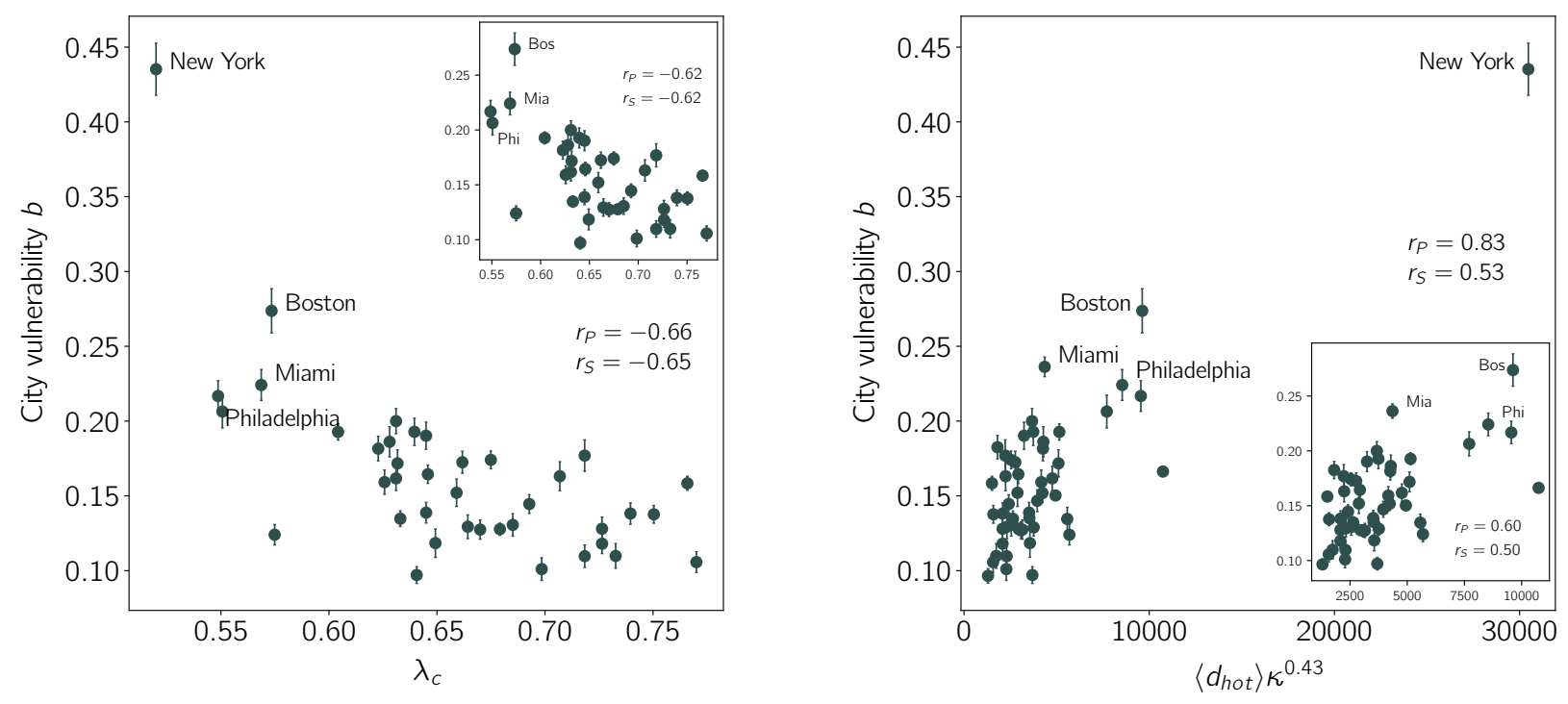

FIG. 3. Validation of the model in US cities A Epidemic growth rate $b$ as a function of the epidemic threshold $\lambda_{c}(p)$ (Eq. S16). In inset we show the same plot after removing NYC. B Epidemic growth rate $b$ as a function of the average population density within hotspots $\left\langle d_{h o t}\right\rangle$ and the hotspot concentration $\kappa$. The exponent 0.43 is introduced to reflect the dependence obtained when fitting the normalized epidemic threshold to $\kappa$ for the United States (Table S2). In both panels, $r_{P}$ and $r_{S}$ denote the Pearson and Spearman rank correlation coefficients among the represented quantities.

Next we connect, the localization of flows to hotspots to the empirical vulnerability in each of the cities. Since $\kappa$ only takes into account flows between hotspots but does not account for their population density, the variable that captures the effective interaction between the residents in hotspots areas should be a combination of both factors. Specifically, we have chosen $\left\langle d_{h o t}\right\rangle \kappa^{0.43}$, where the first term corresponds to the average population density within hotspots and the second term reflects the scaling obtained for the normalized epidemic threshold in the individual case of CBSA from the United States (see Supplementary Section 4 for further details). In Fig. 3B we plot $b$ as a function of this quantity finding a clear monotonically increasing trend. Thus taken together, the results of Fig. 3 indicate that the empirical trends mirror our theoretical formalism, whereby cities that experience strong early growth of the epidemic have a lower threshold, a phenomenon for which one of the main causative mechanisms is that the movement in cities occurs primarily between hotspots. 


\section{E. Potential mitigation measures}

The observations thus far, immediately suggest the possibility of effective mitigation measures that may shore up the robustness of vulnerable cities to the onset of epidemic spread. Given the lack of therapeutics or vaccines for SARS-COV-2, the prevailing strategy adopted globally has been to resort to non-pharmaceutical interventions of which a key ingredient has been aggressive lock-downs. While ostensibly being very effective in mitigating an active epidemic, significant disruption to the socio-economic fabric is one of the unfortunate consequences [58, 59]. Having demonstrated the key role played by the interactions between population density hotspots, we next investigate some targeted interventions, or even preemptions, that are milder than completely restricting mobility city-wide and assess their efficacy in reducing vulnerability. The strategy we pursue is to modify flows between different types of locations in the city without the need to isolate individuals at home. The different schemes are illustrated in the upper panel of Fig. 4. In the first intervention (Intervention I), an asymmetrical strategy involves restricting flows from non-hotspot (heretofore referred to as suburbs) towards hotspot areas and converting them to self-loops, while keeping all other flows the same. Intervention II corresponds to the reverse situation where flows from hotspots to suburbs are converted to self-loops. Finally, in Intervention III, only movement between hotspots is restricted.

For each of these scenarios we recompute the normalized epidemic threshold $\tilde{\lambda}_{c}^{m}(m \in$ $\{$ I,II,III $\}$ ) and plot it against the original threshold, $\tilde{\lambda}_{c}$, in the bottom panels of Fig. 4. In panel $\mathbf{A}$, we find an increase of the threshold across the board indicating that Intervention I is a rather effective strategy. Given that a potential epidemic has a high probability of being seeded and correspondingly spreading extensively in high population density areas, preventing the residents in suburbs from visiting these locations protects them from being exposed to the disease. Conversely in panel $\mathbf{B}$ we see that restricting residents in hotspots to travel to suburbs has the opposite effect in further decreasing the threshold. This counterproductive effect emerges due to a phenomenon discussed in [28]. When there is an asymmetry between the population density in cities, mobility from hotspots to suburbs 
A

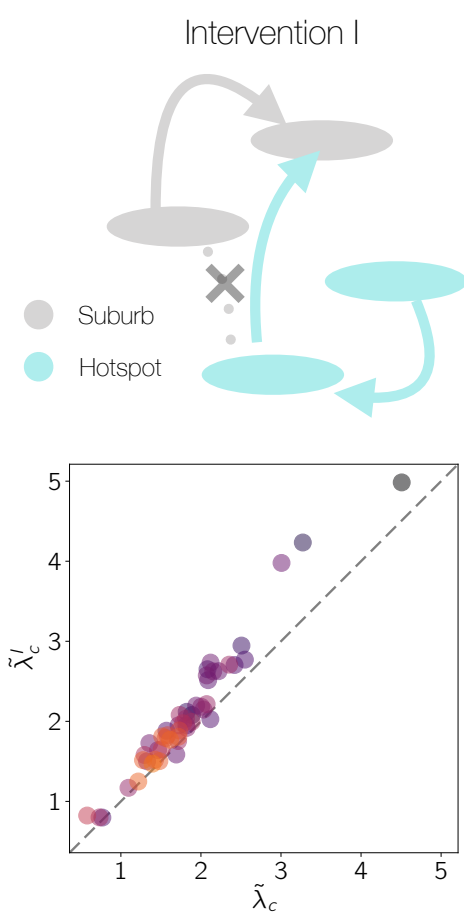

B
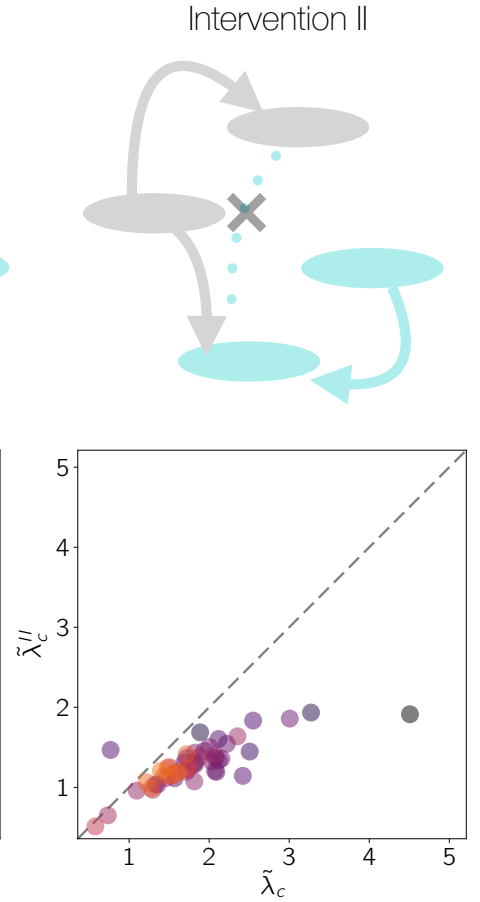

C
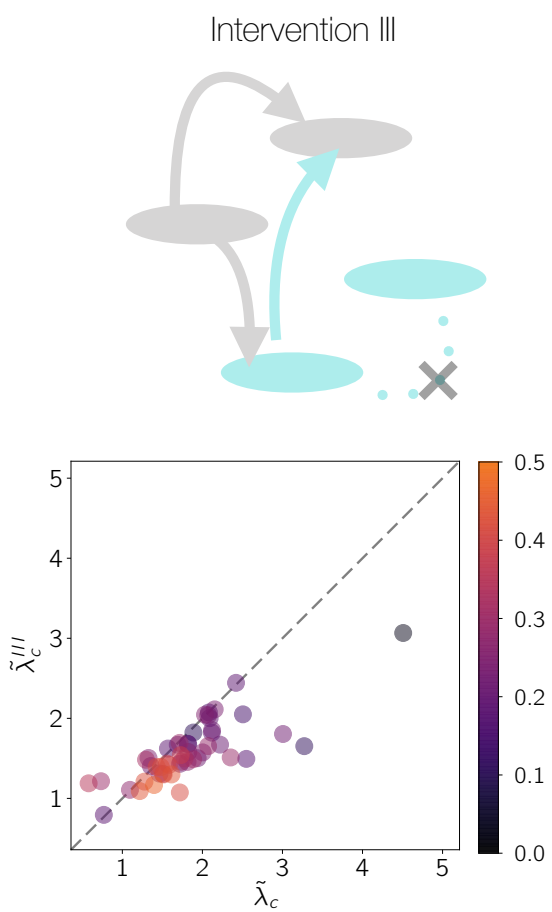

FIG. 4. Impact of mobility interventions on the epidemic threshold within cities in the United States. The impact is quantified by representing the normalized threshold for each intervention, $\tilde{\lambda}_{c}^{I}, \tilde{\lambda}_{c}^{I I}, \tilde{\lambda}_{c}^{I I I}$ (in panels $\mathbf{A}, \mathbf{B}, \mathbf{C}$ respectively) as a function of the normalized threshold $\tilde{\lambda}_{c}$. In all panels, the dashed line denotes the boundary separating the cities for which the intervention is beneficial (above) or detrimental (below). The color of the points represent the hotspot concentration $\kappa$ for each CBSA.

leads to an increase in the threshold due to the dilution of the effective population in the hotspots thus reducing the number of contacts, as well as diverting potentially infectious individuals to to lower density regions where their impact is mitigated. Removing this route makes the situation significantly worse. Finally, panel $\mathbf{C}$ reveals that limiting the mobility between hotspots has a mostly neutral effect, although the trends are noisier given that its effectiveness is closely related to the underlying population density distribution inside hotspots. The results suggest that, among the scenarios outlined, the most beneficial policy is to restrict residents in suburbs from visiting hotspots, while at the same time allowing residents in hotspots to continue with their regular mobility behavior. Note, that several other combinations are possible, for instance a combination of interventions I \& III which is likely to have even more of a beneficial effect. 


\section{Discussion}

Similar to how a virus enters the human body and replicates from cell to cell, the spread of pathogens in susceptible populations is influenced by the interaction between its hosts. Thus, it is the social interactions, mediated by behavioral and mixing patterns, that shapes the spread of disease in human populations. Among these aspects, human mobility is a key factor underlying the unfolding patterns of epidemic outbreaks. Understanding how human mobility shapes the spatiotemporal unfolding of contagious diseases is essential for the design of efficient containment policies to ameliorate their impact. In this paper, we investigate the interplay between the population density and the spatial distribution of flows in urban areas, and its impact on determining exposure to epidemic outbreaks. We report a clear trend worldwide: the existence of a high volume of individuals commuting among the population density centers of a given city makes it more vulnerable to the spread of epidemics. The extent of a city's vulnerability as determined from our formalism, allows us to shed light on a real epidemic scenario: the spread of SARS-COV-2 across United States. In particular, the epidemic threshold determined by the population density profiles and urban mobility patterns provides one of the potential causative mechanisms behind the different levels of infection observed across cities in the United States.

As an application to potential epidemic waves, our indicator allows for identifying those cities that are likely to become epidemic centers once the first imported cases arrive there. This is of importance, given that it can guide authorities to identify places where timely containment policies can be locally implemented to avoid large outbreaks caused by massive community transmission. It is precisely the lack of anticipation to the SARS-COV-2 pandemic, that has led countries to enforce aggressive containment measures aimed at ameliorating the impact of the disease. The predominant strategy has been the implementation of lockdown policies, forcing a large fraction of the population to stay isolated at home, thus reducing considerably their number of interactions. While there is consensus on the effectiveness of these interventions to mitigate an ongoing outbreak, the collateral socio-economic damage caused by lockdowns requires a change of direction towards less-aggressive containment measures. 
In the case of SARS-COV-2, the strict individual isolation characterizing the first interventions have given rise to more relaxed lockdown scenarios combined with efficient Test-Track and Isolate policies [60 62]. Along these lines, we present different realistic scenarios based on modifying mobility habits to actively avoid the emergence of large areas of contagion. Our analysis suggest that a potentially effective policy involves an asymmetric closure of neuralgic centers of the cities, restricting movement to population density hotspots from residents of other areas, while allowing those living in hotspots to commute, in order to dilute the number of contacts in the most vulnerable areas. Teleworking and effective distribution of key services in a city are practical manifestations of such interventions.

An advantage of our formalism is its relative simplicity, paving the way to extend the results to more general scenarios. For example, these results are built upon the assumption that population density centers are much more vulnerable to contagious diseases than scarcely dense areas. While this is a logical assumption, the beneficial effect of mobility from hotspots and suburbs could be reversed for diseases with large reproduction number $R_{0}$. In this scenario, suburbs would also have the potential to develop large outbreaks so the existence of infection routes across the city would lead to an acceleration of the propagation of the epidemic front. Finally, another obvious extension is to consider movement at different geographical scales [63, 64]. Accounting for movement between cities, for instance, could

provide valuable information to coordinate joint efforts among different regions to modify both inter- and intra-urban flows in service of reducing the impact of a pandemic. Needless to say, pandemics are complex processes involving a multitude of spatial and socioeconomic factors. The results presented here may provide one of the building blocks for policy planners in devising effective preventive and mitigation measures for future crises.

\section{Limitations}

These results should be interpreted in light of several important limitations. First, the Google mobility data is limited to smartphone users who have opted in to Googles Location History feature, which is off by default. These data may not be representative of the population as whole, and furthermore their representativeness may vary by location. Importantly, these limited data are only viewed through the lens of differential privacy algorithms, specifically 
designed to protect user anonymity and obscure fine detail. Moreover, comparisons across rather than within locations are only descriptive since these regions can differ in substantial ways.

[1] The world of air transport in 2018. Tech. Rep., International Civil Aviation Organization (2018).

[2] Varghese, N. Globalization of higher education and cross-border student mobility (Citeseer, 2008).

[3] Filatotchev, I., Liu, X., Lu, J. \& Wright, M. Knowledge spillovers through human mobility across national borders: Evidence from zhongguancun science park in china. Research Policy 40, 453-462 (2011).

[4] Williams, A. M. \& Baláž, V. What human capital, which migrants? returned skilled migration to slovakia from the uk 1. International migration review 39, 439-468 (2005).

[5] Boubtane, E., Dumont, J.-C. \& Rault, C. Immigration and economic growth in the oecd countries 1986-2006. Oxford Economic Papers 68, 340-360 (2016).

[6] United Nations, Department of Economic and Social Affairs, Population Division (2019). World Urbanization Prospects 2018: Highlights (ST/ESA/SER.A/421). https:// population.un.org/wup/Publications/Files/WUP2018-Highlights.pdf. Accessed: 201901-30.

[7] Le Néchet, F. Urban spatial structure, daily mobility and energy consumption: a study of 34 european cities. Cybergeo: European Journal of Geography 580 (2012).

[8] Bettencourt, L. M. The origins of scaling in cities. Science 340, 1438-1441 (2013).

[9] Pan, W., Ghoshal, G., Krumme, C., Cebrian, M. \& Pentland, A. Urban characteristics attributable to density-driven tie formation. Nature Communications 4, 1961 (2013).

[10] Kirkley, A., Barbosa, H., Barthelemy, M. \& Ghoshal, G. From the betweenness centrality in street networks to structural invariants in random planar graphs. Nature Communications $\mathbf{9}$, 2501 (2018). 
[11] Van Kerkhove, M. D. et al. Epidemiologic and virologic assessment of the 2009 influenza a (h1n1) pandemic on selected temperate countries in the southern hemisphere: Argentina, australia, chile, new zealand and south africa. Influenza and other respiratory viruses 5, e487-e498 (2011). URL https://pubmed.ncbi.nlm.nih.gov/21668677.

[12] Gomes, M. F. et al. Assessing the international spreading risk associated with the 2014 west african ebola outbreak. PLoS currents 6 (2014).

[13] Zhang, Q. et al. Spread of zika virus in the americas. Proceedings of the National Academy of Sciences 114, E4334-E4343 (2017).

[14] Estrada, E. Covid-19 and sars-cov-2. modeling the present, looking at the future. Physics Reports 869, 1-51 (2020).

[15] Organization, W. H. et al. Coronavirus disease 2019 (covid-19): situation report, 72. Tech. Rep. (2020).

[16] Saunders-Hastings, P. R. \& Krewski, D. Reviewing the history of pandemic influenza: Understanding patterns of emergence and transmission. Pathogens 5, 66 (2016).

[17] Bowen Jr, J. T. \& Laroe, C. Airline networks and the international diffusion of severe acute respiratory syndrome (sars). Geographical Journal 172, 130-144 (2006).

[18] Brockmann, D. \& Helbing, D. The Hidden Geometry of Complex, Network-Driven Contagion Phenomena. Science 342, 1337 LP - 1342 (2013). URL http://science.sciencemag.org/ content/342/6164/1337.abstract.

[19] Colizza, V., Barrat, A., Barthélemy, M. \& Vespignani, A. Predictability and epidemic pathways in global outbreaks of infectious diseases: the sars case study. BMC Medicine 5, 34 (2007). URL https://doi .org/10.1186/1741-7015-5-34.

[20] Lemey, P., Hong, S., Hill, V. \& et al. Accommodating individual travel history and unsampled diversity in bayesian phylogeographic inference of sars-cov-2. Nature Communications 11, $5110(2020)$.

[21] Barbosa, H. et al. Human mobility: Models and applications. Physics Reports 734, 1-74 (2018). URL http://www.sciencedirect.com/science/article/pii/S037015731830022X.

[22] Bonaccorsi, G. et al. Economic and social consequences of human mobility restrictions under covid-19. Proceedings of the National Academy of Sciences 117, 15530-15535 (2020). 
[23] Ahmed, F., Ahmed, N., Pissarides, C. \& Stiglitz, J. Why inequality could spread covid-19. The Lancet Public Health 5, e240 (2020).

[24] Pastor-Satorras, R., Castellano, C., Van Mieghem, P. \& Vespignani, A. Epidemic processes in complex networks. Reviews of Modern Physics 87, 925-979 (2015). URL https://link. aps.org/doi/10.1103/RevModPhys.87.925.

[25] Belik, V., Geisel, T. \& Brockmann, D. Natural Human Mobility Patterns and Spatial Spread of Infectious Diseases. Physical Review X 1, 011001 (2011). URL https://link.aps.org/ doi/10.1103/PhysRevX.1.011001.

[26] Balcan, D. \& Vespignani, A. Phase transitions in contagion processes mediated by recurrent mobility patterns. Nature Physics 7, 581-586 (2011). URL https://doi.org/10.1038/ nphys 1944.

[27] Gómez-Gardeñes, J., Soriano-Paños, D. \& Arenas, A. Critical regimes driven by recurrent mobility patterns of reaction-diffusion processes in networks. Nature Physics 14, 391-395 (2018). URL https://doi.org/10.1038/s41567-017-0022-7.

[28] Soriano-Paños, D., Lotero, L., Arenas, A. \& Gómez-Gardeñes, J. Spreading Processes in Multiplex Metapopulations Containing Different Mobility Networks. Physical Review X 8, 031039 (2018). URL https://link.aps.org/doi/10.1103/PhysRevX.8.031039.

[29] Moss, R., Naghizade, E., Tomko, M. \& Geard, N. What can urban mobility data reveal about the spatial distribution of infection in a single city? BMC Public Health 19, 656 (2019). URL https://doi.org/10.1186/s12889-019-6968-x.

[30] Granell, C. \& Mucha, P. J. Epidemic spreading in localized environments with recurrent mobility patterns. Physical Review E 97, 052302 (2018).

[31] Soriano-Paños, D., Ghoshal, G., Arenas, A. \& Gómez-Gardeñes, J. Impact of temporal scales and recurrent mobility patterns on the unfolding of epidemics. Journal of Statistical Mechanics: Theory and Experiment 2020, 024006 (2020). URL https://iopscience.iop.org/article/ $10.1088 / 1742-5468 / \mathrm{ab} 6 \mathrm{a} 04$.

[32] Tizzoni, M. et al. On the use of human mobility proxies for modeling epidemics. PLOS Computational Biology 10, 1-15 (2014). URL https://doi.org/10.1371/journal.pcbi. 1003716 . 
[33] Mazzoli, M. et al. Field theory for recurrent mobility. Nature Communications 10, 3895 (2019).

[34] Barbosa, H., de Lima-Neto, F. B., Evsukoff, A. \& Menezes, R. The effect of recency to human mobility. EPJ Data Science 4, 21 (2015).

[35] Soriano-Paños, D. et al. Vector-borne epidemics driven by human mobility. Physical Review Research 2, 013312 (2020).

[36] Tizzoni, M. et al. Real-time numerical forecast of global epidemic spreading: case study of 2009 a/h1n1pdm. BMC Medicine 10, 165 (2012). URL https://doi.org/10.1186/ 1741-7015-10-165.

[37] Wesolowski, A. et al. Quantifying the impact of human mobility on malaria. Science 338, 267-270 (2012).

[38] Arenas, A. et al. A mathematical model for the spatiotemporal epidemic spreading of COVID19. medRxiv 2020.03.21.20040022 (2020). URL https://www.medrxiv.org/content/ $10.1101 / 2020.03 .21 .20040022 \mathrm{v} 1$

[39] Costa, G. S., Cota, W. \& Ferreira, S. C. Metapopulation modeling of COVID-19 advancing into the countryside: an analysis of mitigation strategies for Brazil. medRxiv 2020.05.06.20093492 (2020). URL https://www.medrxiv.org/content/10.1101/2020.05.06.20093492v2.

[40] Badr, H. S. et al. Association between mobility patterns and covid-19 transmission in the usa: a mathematical modelling study. The Lancet Infectious Diseases .

[41] Bertuzzo, E. et al. The geography of covid-19 spread in italy and implications for the relaxation of confinement measures. Nature Communications 11, 4264 (2020).

[42] Alirol, E., Getaz, L., Stoll, B., Chappuis, F. \& Loutan, L. Urbanisation and infectious diseases in a globalised world. The Lancet. Infectious diseases 11, 131-141 (2011). URL https: //pubmed.ncbi.nlm.nih.gov/21272793.

[43] Kraemer, M. U. G. et al. Big city, small world: density, contact rates, and transmission of dengue across pakistan. Journal of the Royal Society, Interface 12, 20150468-20150468 (2015). URL https://pubmed.ncbi.nlm.nih.gov/26468065.

[44] Li, R., Richmond, P. \& Roehner, B. M. Effect of population density on epidemics. Physica A: Statistical Mechanics and its Applications 510, 713-724 (2018). URL https://EconPapers. 
repec.org/RePEc:eee:phsmap:v:510:y:2018:i:c:p:713-724

[45] Lee, M., Barbosa, H., Youn, H., Holme, P. \& Ghoshal, G. Morphology of travel routes and the organization of cities. Nature Communications 8, 2229 (2017).

[46] Bassolas, A. et al. Hierarchical organization of urban mobility and its connection with city livability. Nature Communications 10, 4817 (2019). URL https://doi.org/10.1038/ s41467-019-12809-y.

[47] Louail, T. et al. From mobile phone data to the spatial structure of cities. Scientific Reports 4, 5276 (2014). URL https://doi.org/10.1038/srep05276.

[48] Free US Population Density And Unemployment Rate By Zip Code (accessed 2020- 08-27). URL https://blog.splitwise.com/2014/01/06/ free-us-population-density-and-unemployment-rate-by-zip-code/.

[49] Australian Bureau of Statistics. https://www.abs.gov.au/.

[50] Statistics South Africa. http://www.statssa.gov.za.

[51] Facebook (accessed 2020- 08-27). URL https://data.humdata.org/organization/ facebook.

[52] Rader, B. et al. Crowding and the shape of covid-19 epidemics. Nature Medicine (2020).

[53] Di Domenico, L., Pullano, G., Sabbatini, C. E., Boëlle, P.-Y. \& Colizza, V. Impact of lockdown on covid-19 epidemic in île-de-france and possible exit strategies. BMC Medicine 18, 240 (2020).

[54] Prem, K. et al. The effect of control strategies to reduce social mixing on outcomes of the covid-19 epidemic in wuhan, china: a modelling study. The Lancet Public Health (2020).

[55] Gatto, M. et al. Spread and dynamics of the covid-19 epidemic in italy: Effects of emergency containment measures. Proceedings of the National Academy of Sciences 117, 10484-10491 (2020).

[56] https://github.com/nytimes/covid-19-data.

[57] https://usafacts.org/visualizations/coronavirus-covid-19-spread-map/.

[58] Gdp and employment flash estimates for the second quarter of 2020. Tech. Rep., Eurostat (2020).

[59] Gross domestic product, second quarter 2020 (advance estimate) and annual update. Tech. 
Rep., Bureau of Economic Analysis (2020).

[60] Hellewell, J. et al. Feasibility of controlling covid-19 outbreaks by isolation of cases and contacts. The Lancet Global Health (2020).

[61] Ferretti, L. et al. Quantifying sars-cov-2 transmission suggests epidemic control with digital contact tracing. Science 368 (2020).

[62] Salathé, M. et al. Covid-19 epidemic in switzerland: on the importance of testing, contact tracing and isolation. Swiss medical weekly 150, w20225 (2020).

[63] Watts, D. J., Muhamad, R., Medina, D. C. \& Dodds, P. S. Multiscale, resurgent epidemics in a hierarchical metapopulation model. Proceedings of the National Academy of Sciences 102, 11157-11162 (2005).

[64] Balcan, D. et al. Multiscale mobility networks and the spatial spreading of infectious diseases. Proceedings of the National Academy of Sciences 106, 21484-21489 (2009). 


\section{Figures}

Adelaide $(\kappa=0.52)$

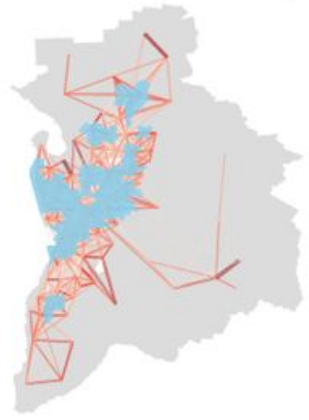

Detroit $(\kappa=0.46)$

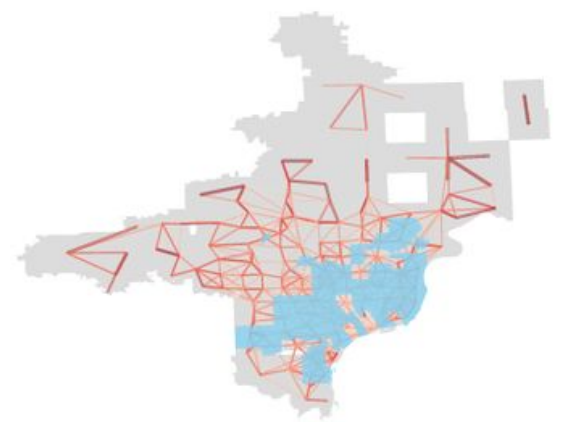

Melbourne $(\kappa=0.25)$

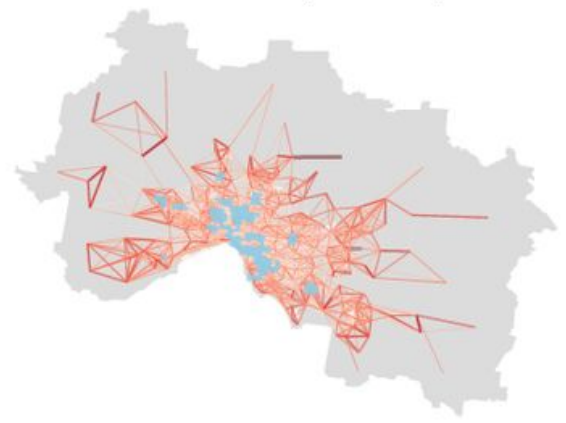

Chicago $(\kappa=0.18)$

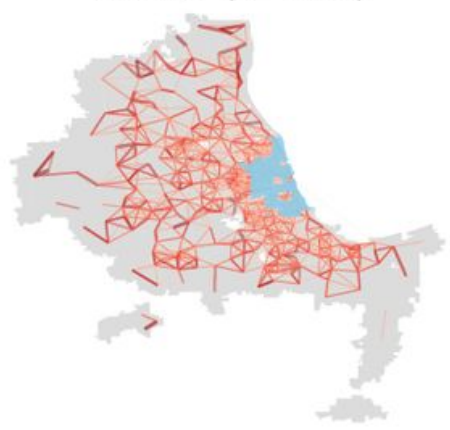

$10^{-2}$
Sydney $(\kappa=0.16)$

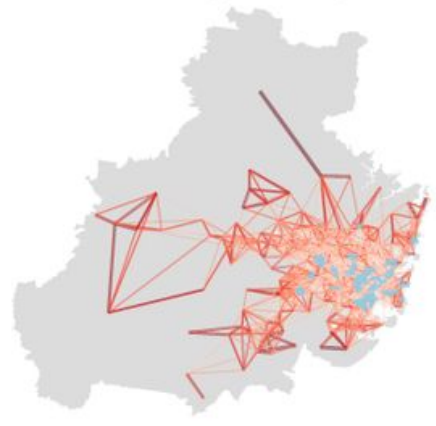

Miami $(\kappa=0.11)$

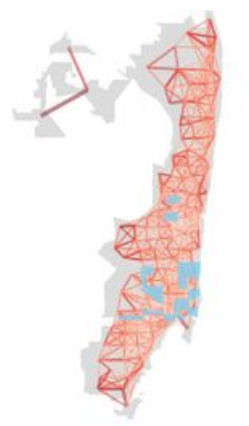

$\begin{array}{llll}10^{-3} & 10^{-2} & 10^{-1} & 10^{0}\end{array}$

\section{Figure 1}

Spatial representation of the urban mobility network for selected cities (upper panel) Autralia, (lower panel) United States. Areas marked blue correspond to population density hotspots. The cities are organized in descending order according to the extent to which mobility ows are concentrated between areas of high population density, k (Eq.(1)). Line color encodes the number of inhabitants following each route normalized by the highest ow observed within each city. 

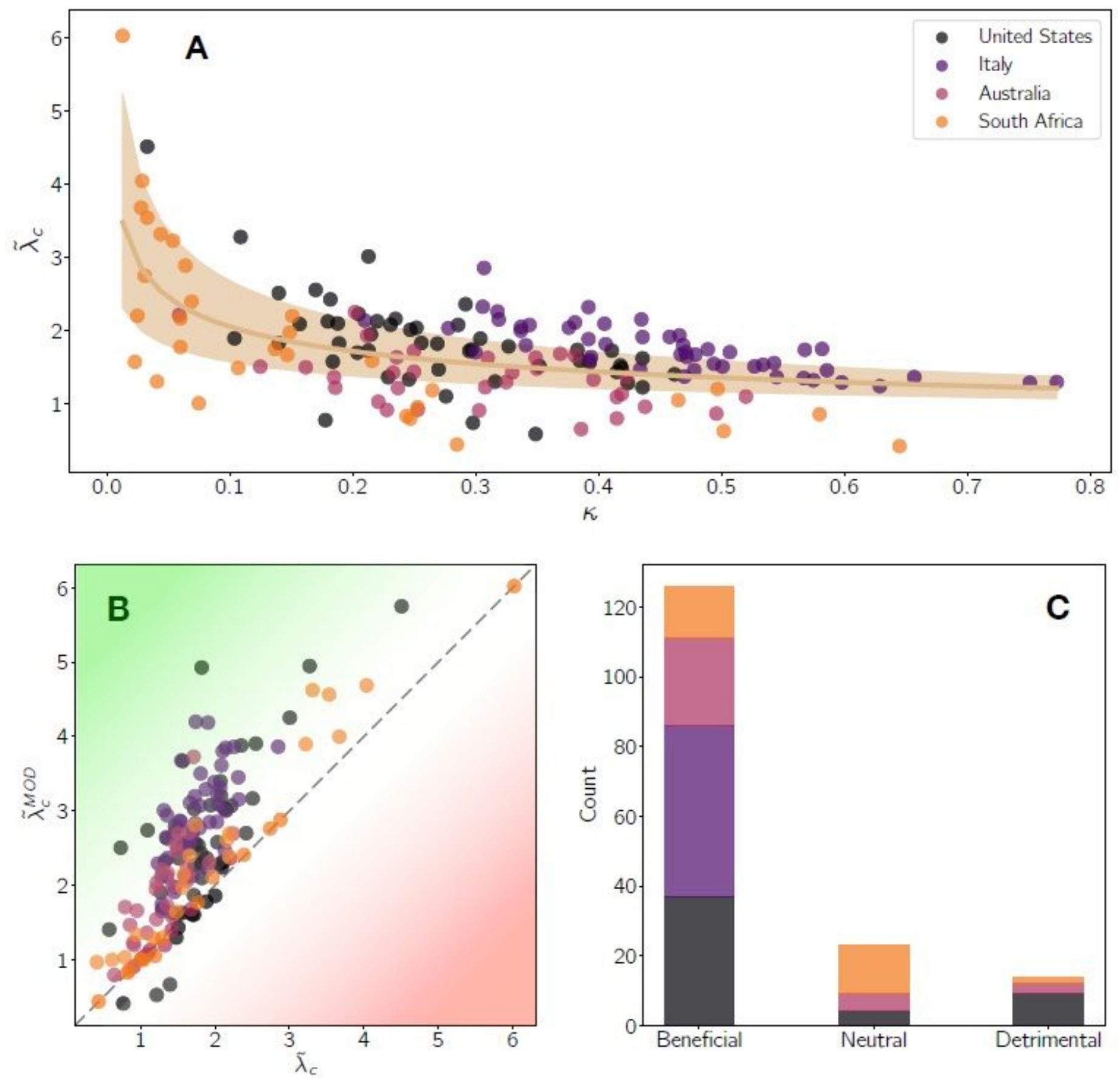

Figure 2

Connecting morphology to vulnerability (see Manuscript file for full figure legend) 

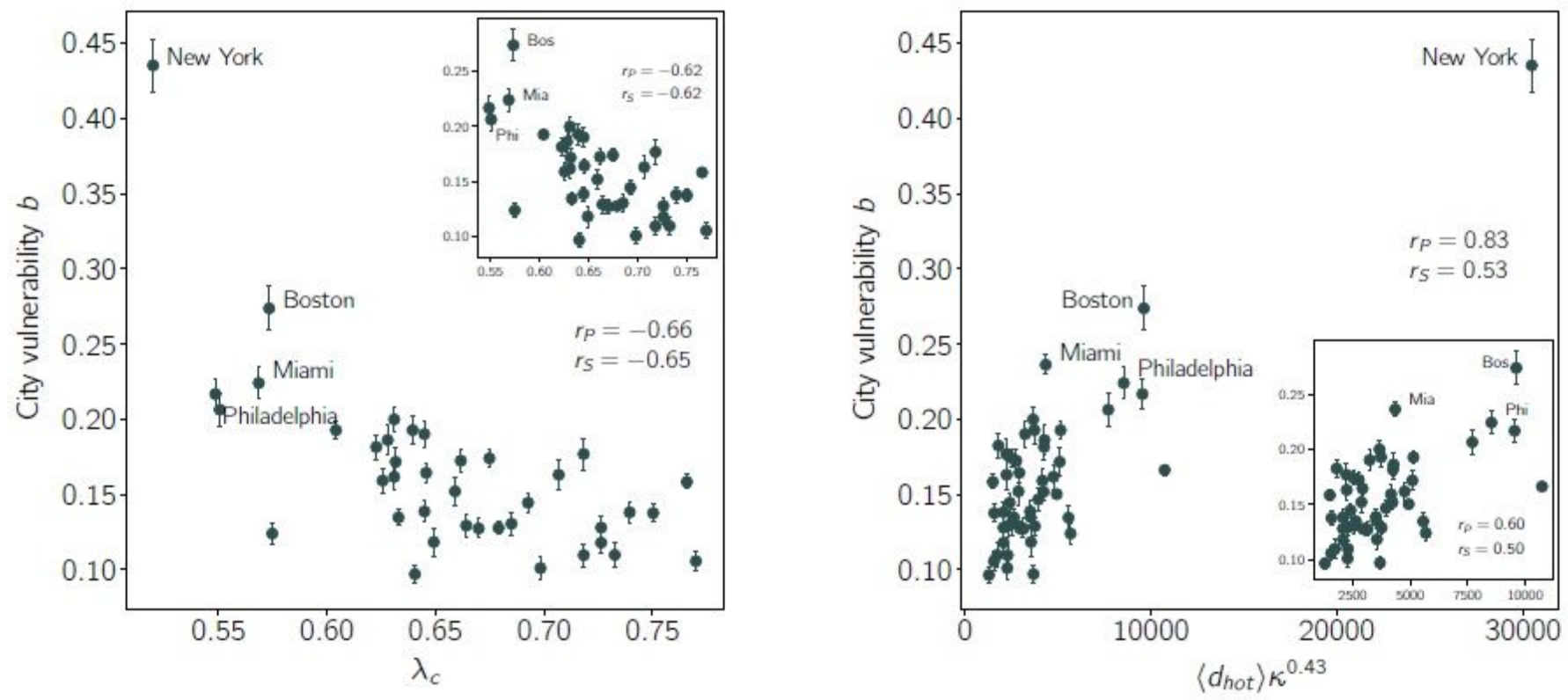

Figure 3

Validation of the model in US cities (see Manuscript file for full figure legend)

A

Intervention |

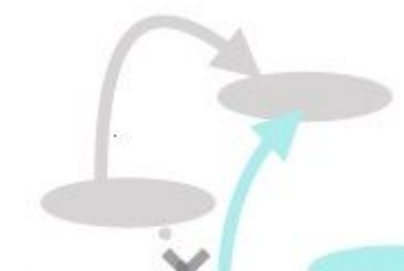

Suburb

Hotspot

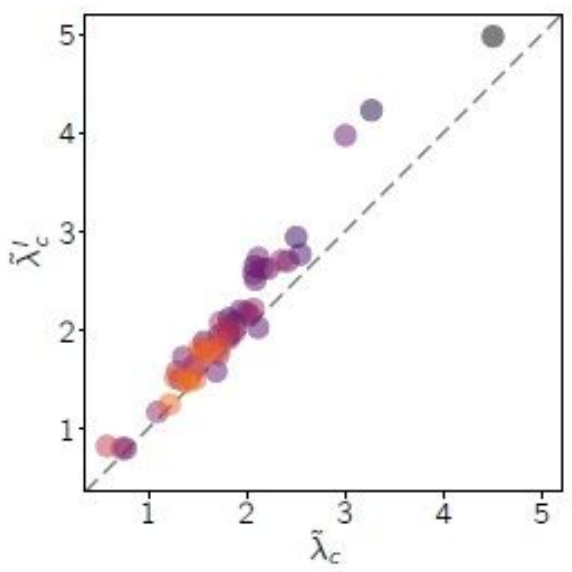

B

Intervention ||
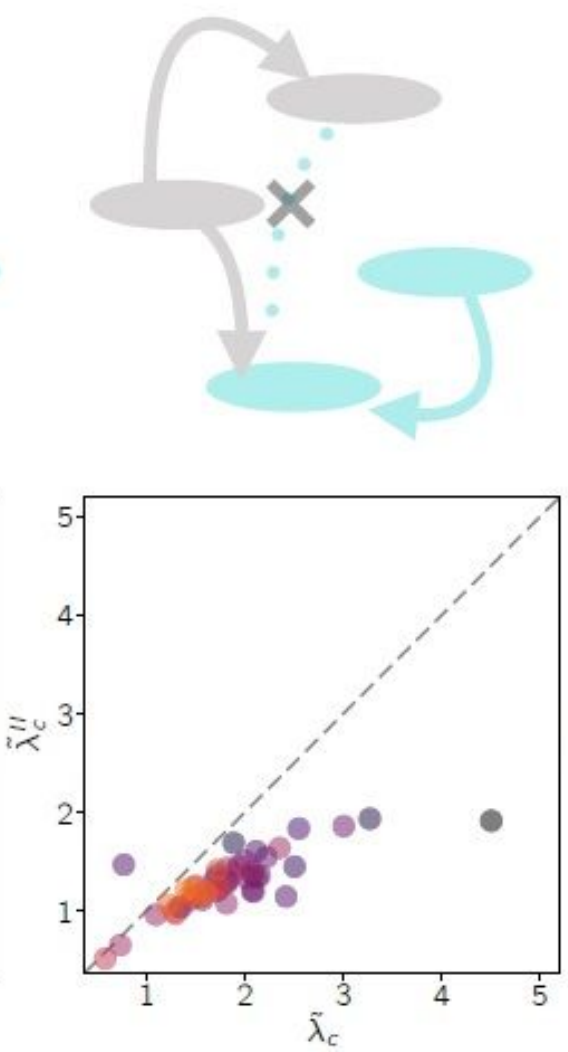

C

Intervention ||
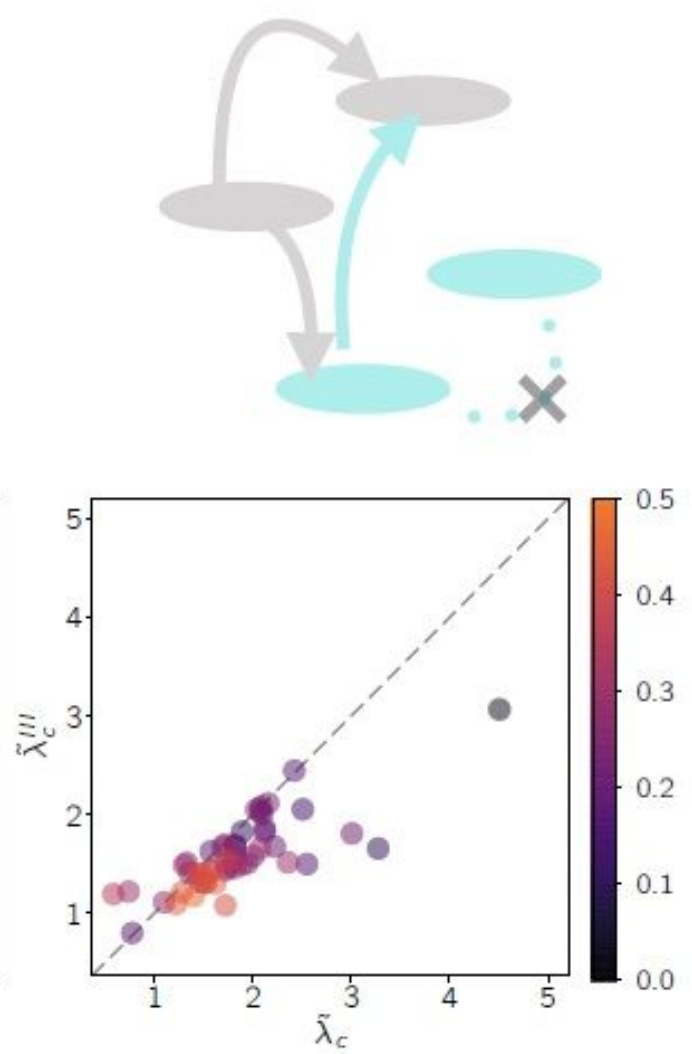
Figure 4

Impact of mobility interventions on the epidemic threshold within cities in the United States.(see Manuscript file for full figure legend)

\section{Supplementary Files}

This is a list of supplementary files associated with this preprint. Click to download.

- SI.pdf 